\title{
The role of animacy in spatial transformations
}

\author{
ALFRED B. YU \\ Washington University, St. Louis, Missouri \\ and Army Research Laboratory, Aberdeen Proving Ground, Maryland \\ AND \\ JEFFREY M. ZACKS \\ Washington University, St. Louis, Missouri
}

\begin{abstract}
We present evidence that different mental spatial transformations are used to reason about three different types of items representing a spectrum of animacy: human bodies, nonhuman animals, and inanimate objects. Participants made two different judgments about rotated figures: handedness judgments ("Is this the left or right side?") and matching judgments ("Are these figures the same?"). Perspective-taking strategies were most prevalent when participants made handedness judgments about human bodies and animals. In contrast, participants generally did not imagine changes in perspective to perform matching judgments. Such results suggest that high-level information about semantic categories, including information about a thing's animacy, can influence how spatial representations are transformed when performing online problem solving. Supplemental materials for this article may be downloaded from http://mc.psychonomic-journals.org/content/supplemental.
\end{abstract}

Spatial perspective taking is the ability to represent aspects of the spatial environment as if from an alternative viewpoint. Perspective taking is useful in a variety of situations, including reasoning about others' goals and intentions (Decety \& Grèzes, 2006; Piaget \& Inhelder, 1956; Vogeley et al., 2004), understanding others' actions (Tversky, Lee, \& Mainwaring, 1999), judging the visibility and relative location of objects (Michelon \& Zacks, 2006), navigation (Kozhevnikov, Motes, Rasch, \& Blajenkova, 2006), and using language (Traxler \& Gernsbacher, 1993). For example, when asked to describe the layout of an apartment, speakers naturally adopt the viewpoint of a hypothetical visitor (Linde \& Labov, 1975). Users of spoken and sign languages ubiquitously perform perspective taking, so that a speaker or signer presents information from the point of view of their conversational partner (Emmorey, Tversky, \& Taylor, 2000; Schober, 1993). Humans sometimes engage in social interactions with other species, such as pets and farm animals (Hare \& Tomasello, 2005), and, in such cases, they may use perspective taking as a means to understand an animal's behavior.

One way in which people may adopt novel perspectives is by imagining that their own body is in a different location, which can be considered to be an imagined spatial transformation. For example, if you wished to tell a friend that they have a smudge on the left side of their face, you could imagine yourself standing in their position and then determine whether the smudge would be on the left or the right side of their face as if it were your own. We will refer to imagining that you are in a different location or orientation as a perspective transformation. Perspective transformations can be distinguished from object-based transformations, in which one imagines that an object is in a different location or orientation. Returning to the example of the friend with a smudge on their left cheek, an object-based transformation strategy for this perspectivetaking task would be to imagine rotating the friend's body into alignment with one's own body. For judgments about others' bodies, this is less intuitive than is the perspective transformation strategy for a majority of observers (Zacks \& Tversky, 2005), but the situation might be different if one were making judgments about a statuette on a desk rather than about a life-sized animate person.

Slightly more formally, both perspective and objectbased transformations are transformations in which the correspondence between spatial reference frames is updated. A spatial reference frame locates things in space. Reference frames differ in the referent with respect to which the target thing is located. Egocentric reference frames locate things relative to one's self. Object-centered reference frames locate things relative to the intrinsic structure of an object. Environmental reference frames locate things relative to the encompassing spatial environment. For example, a viewer looking at the webcam on a laptop computer could describe it as being in front of the viewer (egocentric), at the top of the computer (object centered), or in the corner of the room (environmental). (For a more detailed discussion of spatial reference frames, see McCloskey, 2001.) In a perspective transformation, one's egocentric reference frame is updated relative to the

A. B.Yu, alfredyu@wustl.edu 
environmental reference frame and the object-centered reference frames of particular things in that environment. In an object-based transformation, an object's reference frame is updated relative to the environmental and egocentric reference frames (Zacks \& Michelon, 2005; Zacks \& Tversky, 2005).

In considering spatial problem-solving behavior, it is important to distinguish processes, such as perspective transformations and object-based transformations, from particular tasks. Many tasks that are referred to as perspective-taking tasks can be performed either by performing a perspective transformation or by some alternative transformation (Flavell, Flavell, Green, \& Wilcox, 1981; Michelon \& Zacks, 2006). The term perspective transformation refers to the process, whereas perspective taking refers to the ability to perform a set of tasks. In the literature, the term mental rotation has been used to refer both to particular tasks and to an object-based transformation in which an object-centered reference frame is updated by a rotation. This can lead to confusion, because tasks described as "mental rotation tasks" may not involve mental rotation (although evidence often suggests that they do).

Previous research has indicated that both perspective transformations and object-based transformations are important for spatial reasoning (Zacks, Mires, Tversky, \& Hazeltine, 2000). When instructed to do so, participants can selectively adopt strategies based either on perspective transformations or on object-based transformations (Huttenlocher \& Presson, 1973; Presson, 1982; Wraga, Creem, \& Proffitt, 2000). The two classes of transformation lead to reliably different patterns of response time (RT; Zacks \& Tversky, 2005) and evoked brain activity (Zacks, Ollinger, Sheridan, \& Tversky, 2002; Zacks, Vettel, \& Michelon, 2003). Object-based transformations can be impaired selectively by brain stimulation (Ganis, Keenan, Kosslyn, \& Pascual-Leone, 2000; Harris \& Miniussi, 2003; Zacks, Gilliam, \& Ojemann, 2003), although such selective impairments have not yet been reported for perspective transformations.

In short, a body of evidence dissociates two spatial transformations that can play roles in spatial perspective taking. Some of this research has used a pair of tasks that allow one to compare geometrically equivalent spatial reasoning problems while manipulating the nature of the spatial information to be reported. We describe these tasks in some detail, because they are used in the present experiments.

In the same/different task, participants view pairs of pictures and are asked whether the two pictures depict the same thing. Same pairs are constructed by rotating one of the depicted things relative to the other. Different pairs additionally differ in that the two depicted things differ in some regard; often, they are mirror images of one another (i.e., enantiomorphs). Shepard and Metzler (1971) first described such a task using abstract block figures, finding that greater orientation differences were associated with longer RTs. A large literature has emerged using paradigms derived from the Shepard-Metzler task (Kosslyn, 1980; Shepard \& Cooper, 1982; Zacks, 2008). Often, RTs are found to increase with increasing rotation, and this has been interpreted as evidence that participants are performing an object-based transformation in order to align the reference frame of one object with that of the other (but see Pylyshyn, 2002). In the version of the task used in the present experiments, the pictures are presented one above the other, and the top picture is always presented in an upright orientation, so that the disparity in orientation between the pictures is equivalent to the absolute orientation of the bottom figure (see the right side of Figure 1). In the experiments reported here, all pictures were rotated in the plane of the computer screen.

In the left/right task, participants are asked to determine whether a marker is located on the left or on the right side of a single rotated figure. In the experiments reported here, the stimuli were constructed exactly as the bottom of

\section{A Human Body}

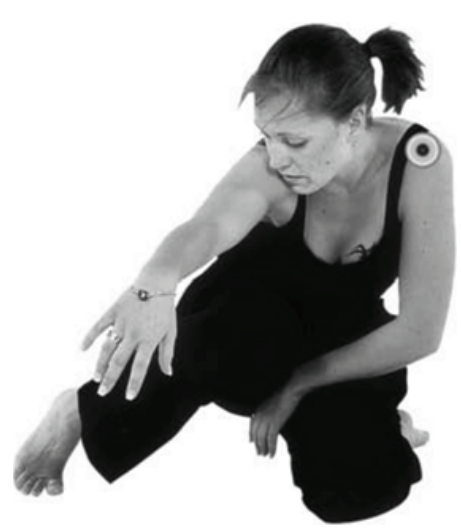

B Animal

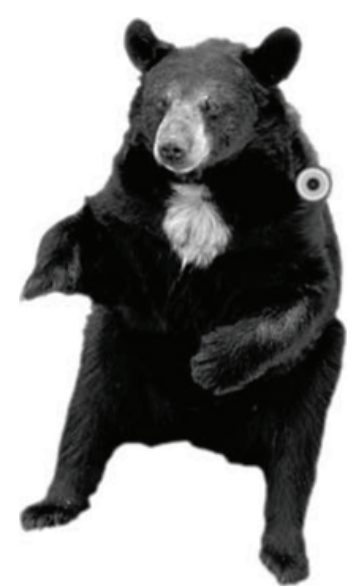

C Inanimate Object

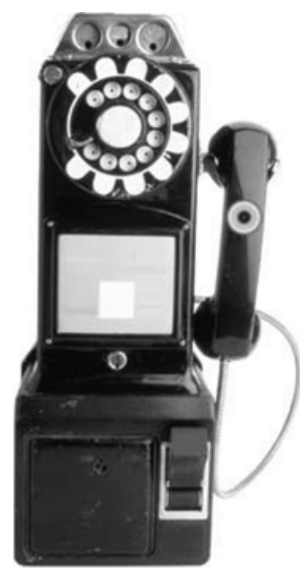

Figure 1. Example stimuli from each of the three item types. Each item was marked on either its left or right side with a multicolored circle. 
the two pictures in the same/different task. Parsons (1987) found that, when viewers performed the left/right task with pictures of human bodies, RTs did not always increase with increasing rotation. Instead, RT functions varied with the plane of rotation and often were nonmonotonic. RT functions for the left/right task closely tracked RT functions for a task in which participants were explicitly instructed to perform a perspective transformation to bring their imagined perspective in line with that of a pictured body. This suggests that, in order to perform the left/right task, participants carried out a perspective transformation. For pictures of bodies that were specifically rotated in the plane of the computer screen and showed bodies facing toward the viewer, RTs were essentially independent of orientation. This provides a useful diagnostic measure of the use of spatial transformation in these task configurations: Object-based transformations should produce increasing RTs with increasing rotation, and perspective transformations should not. This relationship has been verified by several converging approaches in a previous study (Zacks \& Tversky, 2005), which reported that flat RT profiles were observed following explicit instructions to perform perspective transformations, and also in the responses of uninstructed participants who reported using such transformations in postexperiment strategy reports. We wish to emphasize that this dissociation in RT patterns depends on the particular plane in which the stimuli are rotated; for example, for rotations of bodies that faced away from rather than toward the viewer, RTs for imagined perspective transformations and left/right judgments depends strongly on orientation (Parsons, 1987).

\section{Animacy and Spatial Reasoning}

In some circumstances, people perceive images of living things quite differently than they perceive images of nonliving things, such as when viewing rapidly alternating pictures that produce apparent motion (Heptulla-Chatterjee, Freyd, \& Shiffrar, 1996). However, animacy may not consistently affect all spatial judgments. Bryant, Tversky, and Franklin (1992) failed to observe an effect of animacy when participants were asked to judge the relative direction of objects surrounding an upright human actor or an inanimate figure in a memorized scene. It must be noted, however, that the scenes were not presented pictorially, but in the form of narrative texts. This suggests that it may be necessary to distinguish between active viewing and memorybased judgments when it comes to examining the effects of animacy on spatial judgments. An experiment by Zacks and Tversky (2005) compared left/right and same/different judgments using pictorial stimuli representing human bodies and small inanimate objects, such as water pitchers and telephones. By two measures, participants were found to be more likely to use perspective transformations to make left/right judgments about human bodies. First, relations between orientation and RT were affected by stimulus type. The researchers selected a stimulus configuration, based on previously reported findings in the literature, such that object-based transformations would be expected to produce steep increases in RT with increasing orientation, but perspective transformations would not (Parsons, 1987; Zacks,
Rypma, Gabrieli, Tversky, \& Glover, 1999). Left/right judgments about objects showed increases in RT with increasing orientation, whereas left/right judgments about bodies did not. Second, self-report data collected under conditions designed to minimize reactivity showed that participants subjectively experienced perspective transformations more often when making left/right judgments about bodies than when making them about objects.

Within the class of living things, there may be further specialization for the processing of human bodies. Functional MRI and transcranial magnetic stimulation experiments have identified brain regions that are selectively activated when viewing images of bodies (Downing, Jiang, Shuman, \& Kanwisher, 2001; Urgesi, Berlucchi, $\&$ Aglioti, 2004). A small body of previous research suggests that reasoning about human figures differs from reasoning about inanimate objects. Amorim, Isableu, and Jarraya (2006) manipulated the animacy of stimulus figures in a same/different task by adding or subtracting human-like features such as a head, or by constructing the figures so that they represented poses that were either imitable or nonimitable by humans. They found that, with increasingly anthropomorphic figures, RTs became increasingly fast. They interpreted this to reflect a type of spatial perspective taking in which one's own body serves as an analogy for reasoning about the egocentric spaces of other figures.

To date, there has been no evidence of whether nonhuman animals are treated as being similar to humans for spatial perspective taking. Animals share many structural features with humans, including the arrangement of a head and limbs as projections from the body. This structural correspondence may allow for observers to adopt the spatial perspective of animals. In addition, reasoners may be more likely to employ perspective transformations when reasoning about animate agents, because animate agents share conceptual similarities such as intentions and the ability to engage in social behaviors. In this study, we examined participants' tendencies to adopt the spatial perspective when reasoning about human and nonhuman animate figures and about inanimate objects. This extended previous findings in the literature to include nonhuman animals and further allowed us to determine whether perspective taking is a strategy uniquely specialized for making judgments about human figures. We predicted that observers would be unlikely to use perspective transformations to reason about inanimate objects in the left/ right task, but would be more likely to do so when viewing animal figures, as well as humans.

In summary, a variety of studies have shown that living and nonliving things produce distinct behavioral profiles. We proposed that living and nonliving things are treated differently due both to their visual characteristics and to category-level information related to animacy. More specifically, we aimed to determine whether images of nonhuman animals would elicit responses similar to those elicited by images of humans. To the extent that animals are viewed as being similar to humans, we expected to obtain evidence for perspective transformations in the left/ right task. 


\section{EXPERIMENT 1}

In this experiment, we asked participants to perform the left/right and same/different tasks with pictures of bodies, animals, and objects. We hypothesized that participants would be more likely to perform perspective transformations when making left/right judgments about human bodies than when making them about objects, and that they would be more likely to perform object-based transformations when making judgments about objects than when making them about bodies. Following Zacks and Tversky (2005), we designed our experiment so that perspective and object-based transformations should produce different relations between stimulus orientation and RT in the left/right task, allowing us to diagnose which transformation was being performed. As was noted previously, when stimuli are rotated in the picture plane, object-based transformations tend to produce increases in RT with increasing orientation, whereas perspective transformations do not.

\section{Method}

Participants. Forty-four participants from the Washington University Psychology Department's participant pool took part in this experiment for course credit. Data from 6 participants were discarded due to high error rates (greater than $25 \%$ within any block or $15 \%$ overall). Data from the 38 remaining participants ( 24 females; ages 18-22 years) were used for this analysis.

Apparatus. We used PsyScope X (Cohen, MacWhinney, Flatt, \& Provost, 1993) on an Apple Macintosh G3 tower computer to pre- sent stimuli on a 19-in. CRT monitor at a distance of approximately $60 \mathrm{~cm}$. The resolution of the monitor was $1,280 \times 1,024$ pixels. Participant responses were recorded on a USB button box (ioLab Systems).

Stimuli. Photographs were selected from commercial stock art libraries and the World-Wide Web and depicted a broad assortment of human bodies, nonhuman animals, and inanimate objects. Pictures were cropped from their background if necessary and were arranged so that the centroid of their silhouette was centered on a white background. We used 24 images of each type (human body, nonhuman animal, inanimate object; see Figure 2 for examples of each). Natural images from these categories differ on low-level visual characteristics, including symmetry and skinniness (ratio of vertical to horizontal extent). We selected images to provide overlapping distributions on these characteristics, but not to eliminate differences between categories entirely. The supplemental material contains a more in-depth discussion of these two visual characteristics and their effects on task performance.

In order to unambiguously mark the left or right side, an image of a small multicolored circle was placed on a prominent side of each depicted body, animal, or object. Images were scaled to fit within an $11-\mathrm{cm}$ square and occluded approximately $9^{\circ}$ of visual angle. For each original image, a mirror-image version was created by reflecting it about its vertical axis. Rotated images were obtained by rotating each image through successive multiples of $30^{\circ}$ (ranging from $30^{\circ}$ to $330^{\circ}$ ).

Tasks. Participants performed the left/right and same/different tasks on the computer (see Figure 1). A fixation cross was presented for $1,000 \mathrm{msec}$ at the start of each trial. In the left/right task, a single stimulus was then presented at the center of the screen at a randomly chosen orientation, and participants were asked to identify whether the colored circle was on the left or right side of the body, animal, or object. In the same/different task, two images were presented si-

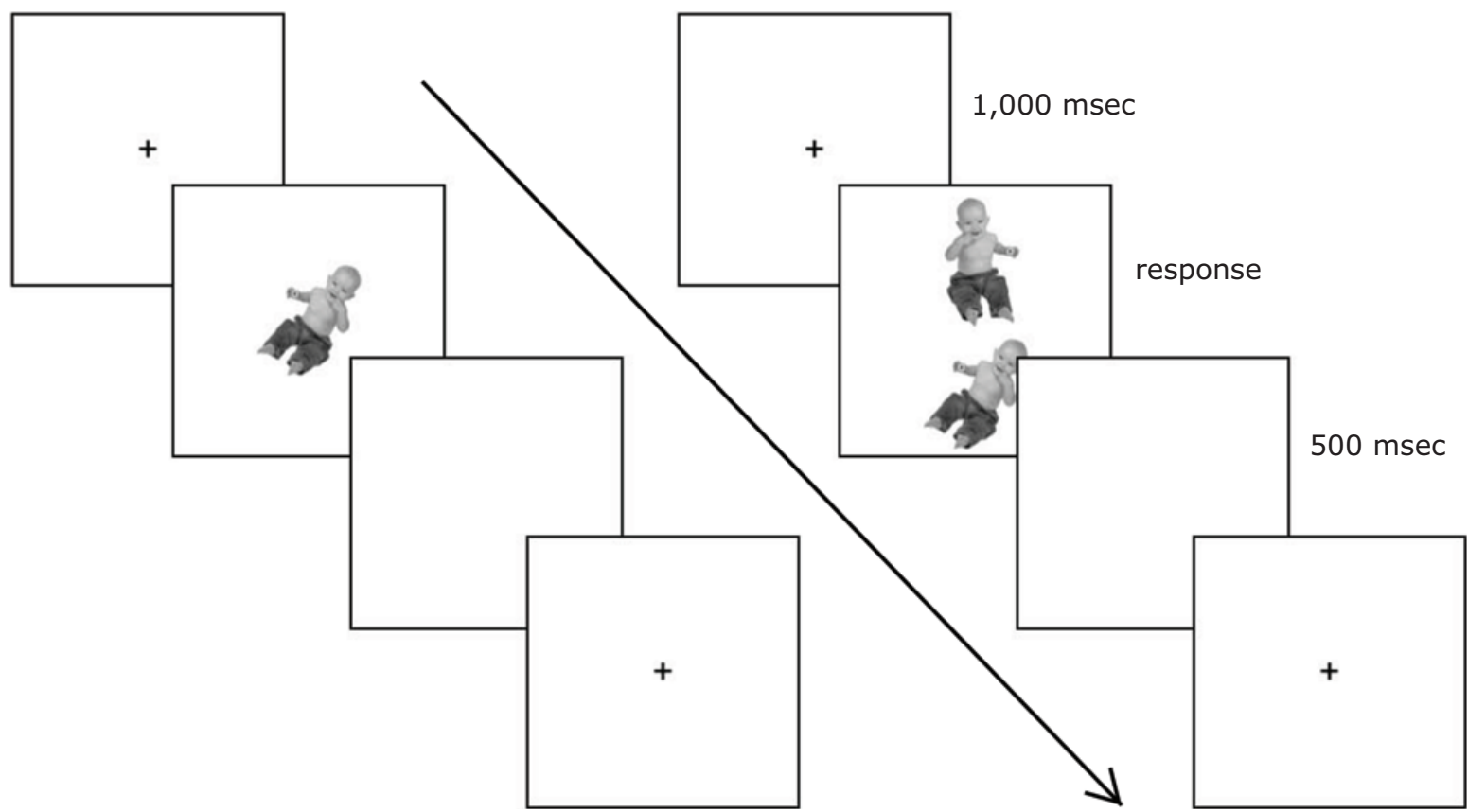

Figure 2. Timeline of (A) the left/right task and (B) the same/different task. In the left/right task, the marker is on the baby's right hand, meaning that, in this example, the participant was to respond by pressing the button marked "Right." In the same/different task, the marker is on the left hand in the top image and on the right hand in the bottom image, meaning that, in this example, the participant was to respond by pressing the button marked "Different." Both trials depict a stimulus with a $30^{\circ}$ orientation disparity. 
multaneously, with the top image upright and the bottom image at a randomly chosen orientation. Participants were asked to determine whether two figures were identical or mirror-flipped versions of one another. In both tasks, participants were asked to respond as quickly as possible while remaining accurate. At the beginning of the experiment, participants were given two practice blocks to practice making each of these judgments. Each practice block consisted of 12 trials of a single task, with items from each of the three groups pseudorandomly intermixed. Each of the six blocks consisted of 96 trials of a single task in the nonpractice blocks, meaning that the full experiment consisted of 24 practice trials and 576 experimental trials. Participants performed a block of one task and then a block of the other for a single item type before moving on to the next item type in the next two blocks. Both task order and item type order were fully counterbalanced across participants. Following the computer tasks, participants filled out a questionnaire that asked them to describe the strategies that they used during each block of the experiment.

\section{Results}

After exclusion of 6 participants with high error rates, overall error rates ranged from $0.9 \%$ to $12.3 \%$, and perblock error rates ranged from $0 \%$ to $17.7 \%$. RTs were trimmed by discarding error trials, values less than $300 \mathrm{msec}$, and values greater than $3 S D$ s above the mean for each combination of participant and task. Several participants indicated that they did not understand the canonical orientation of one particular inanimate object (an old-fashioned camera). We discarded this item for two reasons: RTs were much greater than the mean for all other items $(2,465$ vs. $1,588 \mathrm{msec})$, and the error rate was much greater $(36.9 \%$ vs. $4.9 \%)$. A combined total of $7.4 \%$ of all trials were removed from further analysis. Clockwise and counterclockwise rotations were collapsed for analysis. This practice is supported by the finding that participants tend to compare pairs of images by imagining the rotation of one image along the shortest geometric path (Shepard, 1988) and by the observation that RTs to both clockwise and counterclockwise rotations increased symmetrically with increasing orientation in both tasks.

RT analysis. For the first section of this analysis, we collapsed across stimulus images within each item type and calculated the mean RT for each combination of participant, task, item type, and orientation. These mean RTs were then submitted to a within-participants 2 (task) $\times$ 3 (type) $\times 7$ (orientation) repeated measures ANOVA. As can be seen in Figure 3, mean RTs were lower overall in the left/right task when compared with those in the same/ different task [main effect of task, $F(1,37)=1,301, p<$ $.001]$. There was an overall increase in RT with increasing rotation [main effect of orientation, $F(6,222)=42.0, p<$ $.001]$. Collapsing across both tasks, responses to images depicting human bodies were fastest, whereas RTs for images of inanimate objects were slowest; those for images of animals were intermediate [main effect of item type, $F(2,74)=4.0, p=.022]$. RTs in the same/different task increased with increasing orientation more than those in the left/right task [task $\times$ orientation, $F(6,222)=17.2$, $p<.001]$. The two-way interactions involving item type were not significant [task $\times$ item type, $F(2,74)=0.9, p=$ .393 ; orientation $\times$ item type, $F(12,444)=0.5, p=.912]$.

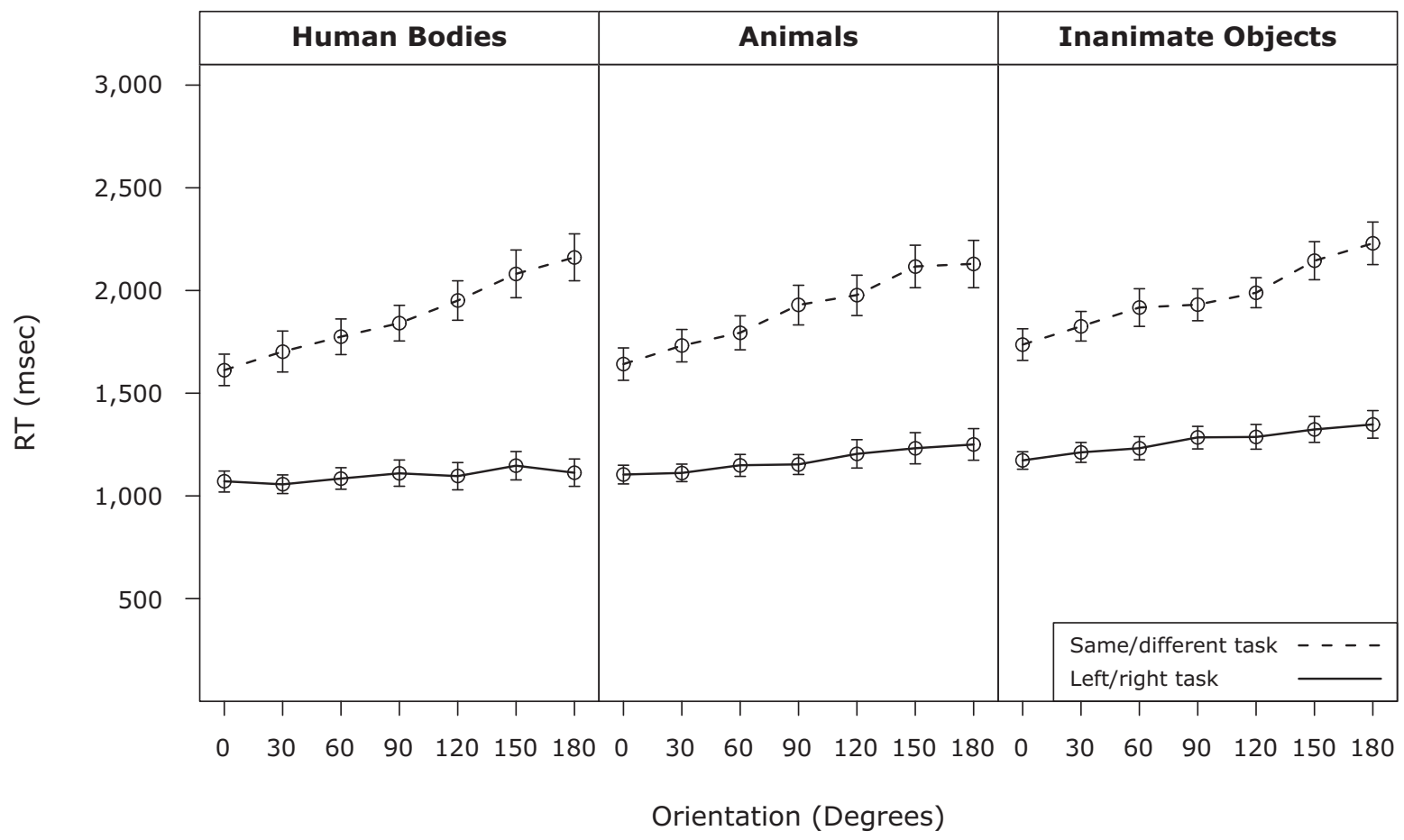

Figure 3. Mean response times (RTs) from Experiment 1 for both the left/right task and same/different task plotted against orientation disparity (abscissa). Each panel represents data from one of the item types. Error bars represent the standard error of the mean across participants. 


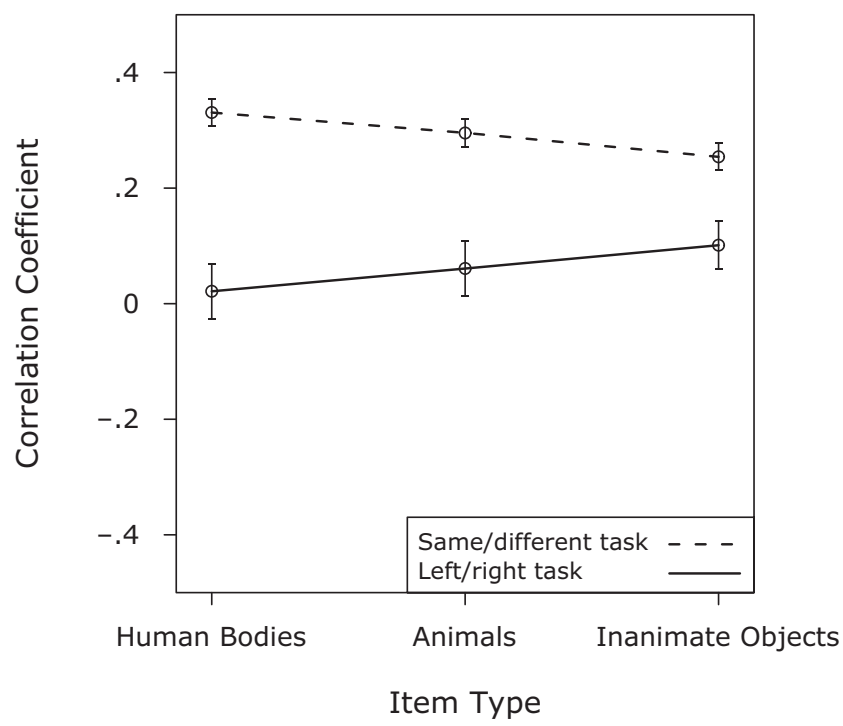

Figure 4. Mean correlation coefficients for each combination of task and item type in Experiment 1. Error bars represent the standard error of the mean across participants.

Finally, there was a significant three-way task $\times$ type $\times$ orientation interaction $[F(12,444)=2.1, p=.017]$. The nature of this interaction was such that the difference in slopes between the left/right and same/different tasks was greatest for images depicting human bodies and smallest for those depicting inanimate objects.

To better characterize the three-way interaction, we ran separate type $\times$ orientation ANOVAs for the left/right and same/different tasks. For neither task was the type $X$ orientation interaction statistically significant [left/right task, $F(12,444)=1.2, p=.249$; same/different task, $F(12,444)=1.3, p=.238]$. Thus, although the pattern of the three-way interaction was consistent with the hypothesis that item type affects spatial transformation strategy in the left/right task, this latter analysis did not provide strong statistical support.

Correlations between orientation and RT. To provide a more focused test of our hypothesis about the effect of task and item type on RT patterns, we analyzed the correlations between RT and orientation for each combination of item type and task. Increases in RT with increasing orientation produce positive correlations, whereas responses that are independent of orientation produce correlations near zero. Thus, for this stimulus configuration, positive correlations are associated with object-based transformations, and zero correlations are associated with perspective transformations. Correlation coefficients were averaged across participants and are plotted in Figure 4 . The mean correlation coefficients for the left/right task did not differ significantly from zero for stimuli depicting bodies $[t(37)=0.452, p=.654]$ or animals $[t(37)=1.30$, $p=.203]$. However, the mean correlation coefficient was significantly different from zero for the stimuli depicting inanimate objects in the left/right task $[t(37)=2.44$, $p=.020]$ and for all item types in the same/different task (smallest $t=11.0, p<.001$ ). This replicates a finding in a similar experiment comparing responses to stimuli depicting human bodies and inanimate objects (Zacks \& Tversky, 2005).

To examine the effects of task and type on the correlation coefficients, the data were submitted to a withinparticipants 2 (task) $\times 3$ (type) ANOVA. The dependent variable was the correlation coefficient between RT and orientation, calculated for each combination of participant, task, and type. Correlation coefficients were greater for the same/different task [main effect of task, $F(1,37)=29.5, p<.001]$. Overall, correlation coefficients did not differ between item types [main effect of item type, $F(2,74)=0.006, p=.995]$. However, item type interacted with task $[F(2,74)=7.3, p=.001]$, such that the greatest difference between the two tasks was observed for stimuli depicting bodies; the smallest difference was observed for those depicting inanimate objects.

We followed up the ANOVA with separate planned comparisons between each pair of item types within each task, correcting for multiple comparisons by using Tukey's HSD. In the left/right task, correlations were significantly smaller for pictures of human bodies $(M=.02, S D=.29)$ than for pictures of inanimate objects $(M=.10, S D=$ .26). For the same/different task, correlations were significantly greater for pictures of bodies $(M=.33, S D=.14)$ than for pictures of objects $(M=.02, S D=.29)$.

Introspective strategy reports. Two experimenters scored each exit questionnaire by assessing the degree to which each response corresponded to one of two predefined strategies. The first strategy was "imagining a picture moving," which represented an object-based 
Table 1

Frequencies of Each Self-Reported Strategy in Experiment 1

\begin{tabular}{clccc}
\hline & & \multicolumn{3}{c}{ Transformation Type } \\
\cline { 3 - 5 } \multicolumn{1}{c}{ Task } & \multicolumn{1}{c}{$\begin{array}{c}\text { Object- } \\
\text { Based }\end{array}$} & Perspective & $\begin{array}{c}\text { Both or } \\
\text { Neither }\end{array}$ \\
\hline Same/different & Human bodies & $30(78.9 \%)$ & $3(7.9 \%)$ & $5(13.2 \%)$ \\
& Animals & $32(84.2 \%)$ & $5(13.2 \%)$ & $1(2.6 \%)$ \\
& Inanimate objects & $29(76.3 \%)$ & $6(15.8 \%)$ & $3(7.9 \%)$ \\
\multirow{5}{*}{ Left/right } & Human bodies & $13(34.2 \%)$ & $15(39.5 \%)$ & $10(26.3 \%)$ \\
& Animals & $12(31.6 \%)$ & $23(60.5 \%)$ & $3(7.9 \%)$ \\
& Inanimate objects & $14(36.8 \%)$ & $21(55.3 \%)$ & $3(7.9 \%)$ \\
\hline
\end{tabular}

Note-Percentages are given in parentheses. Row totals sum to $100 \%$.

transformation strategy. The second strategy was "imagining myself moving or imagining a change in my own perspective," which represented a perspective transformation strategy. All responses that did not fit into the previous two categories were labeled "other." Responses could belong to more than one category. Consensus was reached through discussion in each case of rater disagreement, which occurred for $1.8 \%$ of the total responses. The frequencies of occurrence for each strategy are presented in Table 1. Four participants made a total of 8 responses (3.5\% of all participants' responses) that were categorized as representing both object-based and perspective transformations. These responses were not used in the subsequent analyses. Seventeen $(7.5 \%)$ of all responses were scored only as "other" responses. A majority of participants $(25$ out of 38) reported using object-based transformations for all three item types during the same/different task. In the left/right task, participants reported using object-based transformations and perspective transformations with approximately equal frequency. This latter result was contrary to our predictions.

The introspective strategy data along with the behavioral measures of task performance suggest that participants used object-based transformation strategies when they performed the same/different task. There was a larger variety of self-reported strategies when participants performed the left/right task.

\section{Discussion}

We found evidence for distinct RT patterns for items differing in animacy in two spatial tasks. In the left/ right task, RTs to stimuli depicting bodies were largely independent of orientation. As indicated by the correlation coefficient analysis, RTs to stimuli depicting objects increased significantly with increasing orientation. This pattern suggests that participants were more likely to use perspective transformations to perform the left/right task with bodies than with objects. We note that the difference was somewhat smaller than that reported by Zacks and Tversky (2005).

Positive correlation coefficients indicated that RTs in the same/different task increased with increasing stimulus orientation, for both body and object stimuli. This pattern suggests that participants predominantly used objectbased transformations to perform the same/different task, for both body and object stimuli. Unexpectedly, responses to images of human bodies were slightly, but significantly, more orientation dependent than were responses to images of objects. In a previous study comparing pictures of bodies with pictures depicting abstract forms derived from Shepard and Metzler (1971) stimuli, Amorim et al. (2006) found the opposite pattern: Bodies produced shallower slopes than did nonbody objects. In future studies, it will be important to assess the replicability and generalization of the present result.

For both tasks, the pattern of RTs for animals fell numerically between those for bodies and for objects. One interpretation of this pattern is that participants adopted consistent strategies within each block and that their strategy when viewing animals happened to produce intermediate RTs. Another possibility is that individual participants adopted mixed strategies when viewing stimuli depicting animals, at times treating them as human bodies and at other times treating them as objects. However, participants rarely reported such mixed strategies explicitly on the postexperimental questionnaire. The experimental design and analyses used here do not allow us to discriminate between these possibilities.

Unlike the behavioral measures, introspective strategy reports did not reflect a more frequent usage of perspective transformations when participants viewed stimuli depicting human bodies or animals. It may have been difficult for participants to remember differences in strategy use across the six experimental blocks, especially since the task alternated between each block. In Experiment 2 we tested each participant on only one of the two spatial reasoning tasks, both to replicate the basic findings from Experiment 1 and to reduce the possibility that strategy reports would be contaminated by carryover effects.

\section{EXPERIMENT 2}

Experiment 2 was similar to Experiment 1, except that task was manipulated between rather than within participants. This was done to eliminate carryover effects of task and to reduce the possibility of demand characteristics influencing the strategy reports. Each participant performed either the left/right task or the same/different task with pictures of bodies, animals, and objects. The number of participants was increased to counteract the reduced statistical power when conducting a between-participants manipulation. 


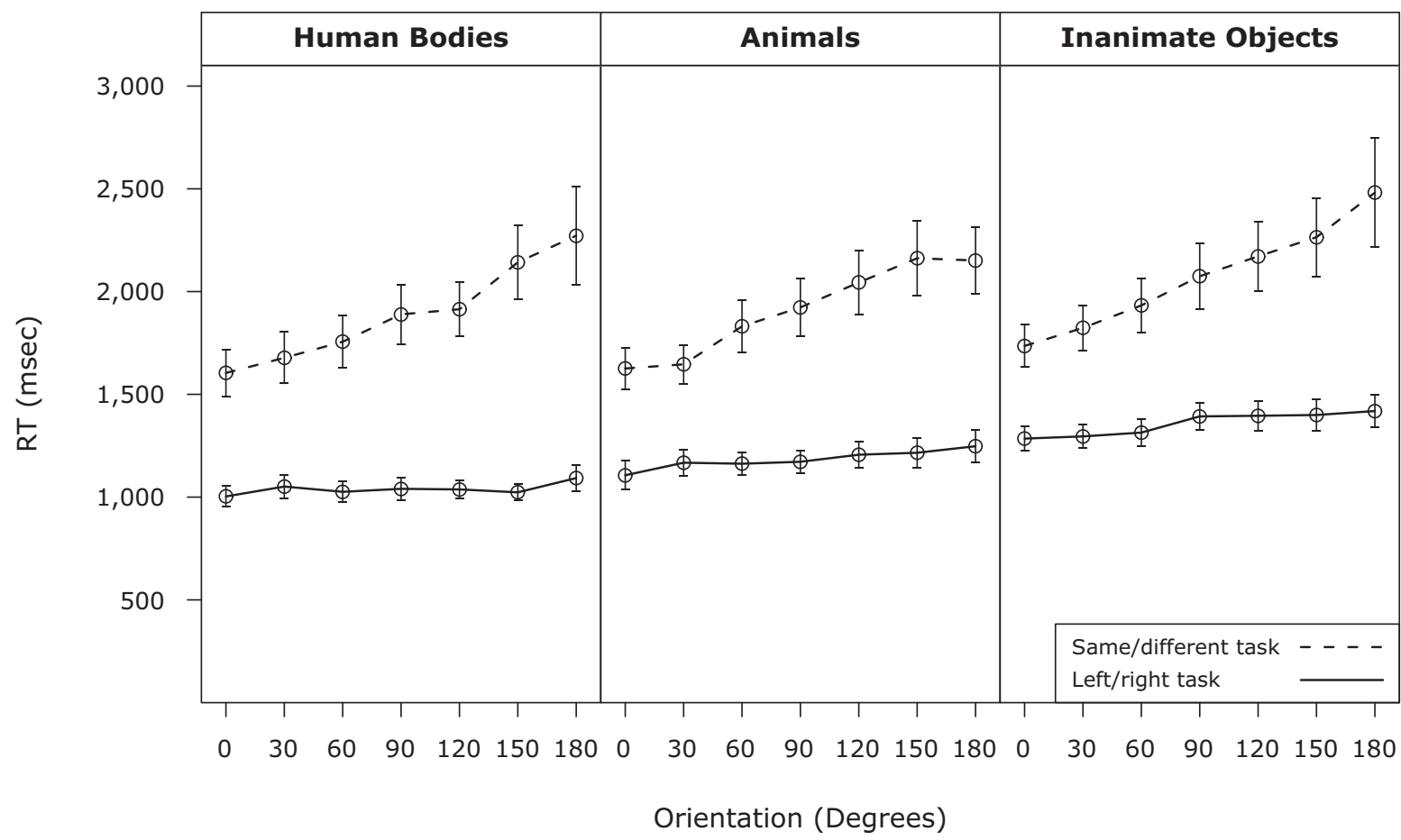

Figure 5. Mean response times (RTs) from Experiment 2 for both the left/right task and same/different task plotted against orientation disparity (abscissa). Each panel represents data from one of the item types. Error bars represent the standard error of the mean across participants.

\section{Method}

Participants. Fifty participants from the Washington University Psychology Department's participant pool took part in this experiment for course credit. The data from 2 participants were removed from further analysis because they had an error rate greater than $15 \%$ on any individual block. Of the 48 remaining participants, 29 were female, and their ages ranged from 18 to 22 years.

Apparatus and Stimuli. The experimental apparatus and stimuli were identical to those in Experiment 1.

Tasks and Procedure. The tasks were the same as those used for Experiment 1, with the exception that participants performed only one of the two possible tasks. Each of the three blocks consisted of 96 trials of a single item type. There were 288 trials in all for each participant.

\section{Results}

The data were trimmed, as was done in Experiment 1 (3.1\% of all trials were removed). The RTs from Experiment 2 are plotted in Figure 5. The overall pattern of results was quite comparable to that from Experiment 1.

RT analysis. We performed a 2 (task) $\times 3$ (type) $\times$ 7 (orientation) ANOVA on RT data collapsing across the stimuli of each item type. RTs were greater in the same/ different task [main effect of task, $F(1,46)=26.6, p<$ .001 ] and were greater for increasingly rotated items [main effect of orientation, $F(6,276)=20.5, p<.001]$. Overall, RTs to stimuli depicting bodies were fastest, and those depicting objects were slowest [main effect of type, $F(2,92)=$ $31.0, p<.001]$. The effect of orientation depended on the task being performed, with RTs for the same/different task being more orientation dependent [task $\times$ orientation,
$F(6,276)=20.5, p<.001]$. The task $\times$ item type interaction was marginally significant $[F(2,92)=3.0, p=.057]$; same/different task RTs appeared to be similar across the three item types, whereas left/right task responses to human body stimuli appeared to be faster than those for stimuli depicting animals or objects. Unlike Experiment 1-and contrary to our expectations - the three-way task $\times$ type $\times$ orientation interaction was not significant $[F(12,552)=$ $0.997, p=.45]$. This may reflect the smaller power to detect differences when using a between-participants manipulation of the experimental task, or it may indicate that participants tended to use the same strategy across all item types due to carryover effects (because item type was still manipulated within participants). We ruled out this explanation by failing to find interactions between item type order and any other factor in a 2 (task) $\times 3$ (item type) $\times 7$ (orientation) $\times 6$ (item type order) ANOVA on RT.

Correlations between orientation and RT. As in Experiment 1, we used each participant's correlation between RT and orientation as another dependent measure. The mean correlation values across participants for each task and item type are presented in Figure 6. As can be seen in the figure, left/right task correlation values for stimuli depicting human bodies were close to zero, correlations for stimuli depicting inanimate objects were positive, and correlations for stimuli depicting animals were intermediate. In the same/different task, correlations were all well above zero, were smallest for inanimate objects, and were largest for human bodies. 


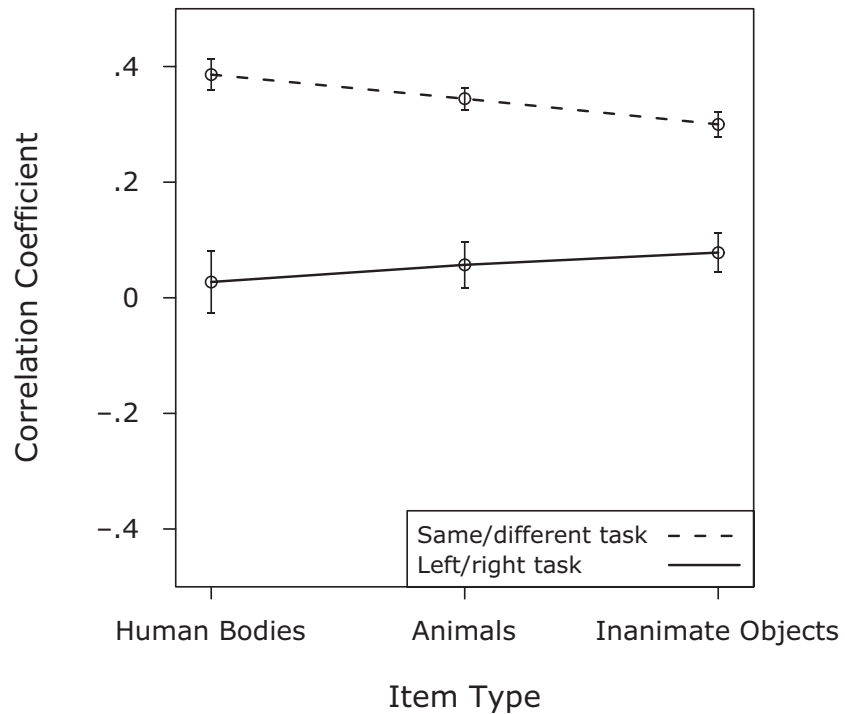

Figure 6. Mean correlation coefficients for each combination of task and item type in Experiment 1. Error bars represent the standard error of the mean across participants.

The correlation coefficients were submitted to a 2 (task) $\times 3$ (type) ANOVA, with task as a betweenparticipants factor and item type as a within-participants factor. Correlation coefficients were greater for the same/ different task [main effect of task, $F(1,46)=48.2, p<$ $.001]$. The between-task difference in correlation coefficients was greatest for stimuli depicting human bodies and smallest for those depicting inanimate objects [task $\times$ item type interaction, $F(2,92)=4.7, p<.05]$. We examined each of the two tasks in isolation by using separate one-way ANOVA models, with item type as the single factor. For participants who performed the left/right task, correlation coefficients did not differ across item types $[F(2,46)=1.0, p=.394]$. However, for participants who performed the same/different task, there was a significant main effect of type $[F(2,46)=6.0, p<.01]$. Post hoc comparisons using Tukey's HSD indicate that the correlation coefficients differed between images of human bodies $(M=.39, S D=.13)$ and images of inanimate objects $(M=.30, S D=.11)$ at $p<.05$. Correlation coefficients did not differ between images of human bodies and images of animals $(M=.34, S D=.09)$, nor did they dif- fer between images of animals and images of inanimate objects. The correlation coefficients for the left/right task were more variable across participants than were those in the same/different task, which could explain the lack of a significant type effect in the separate analysis of the left/ right task data.

Introspective strategy reports. The frequencies of occurrence for each self-reported strategy are presented in Table 2. Eight participants made a total of nine responses (6.1\% of all participants' responses) that were categorized as representing both object-based and perspective transformations. These responses were not used in the subsequent analyses. Twelve $(8.2 \%)$ of the total responses were scored only as "other" responses and were also removed from further analyses. Most participants reported using object-based transformations during the same/different task. In the left/right task, participants reported using perspective transformations with greater frequency than they used the other strategies. Of the 24 participants, 19 reported using object-based transformations across all three blocks in the same/different task, whereas only 5 reported using such transformations across all blocks of the left/

Table 2

Frequencies of Each Self-Reported Strategy in Experiment 2

\begin{tabular}{clrcc}
\hline \multirow{2}{*}{ Task } & \multicolumn{3}{c}{ Transformation Type } \\
\cline { 3 - 5 } & \multicolumn{1}{c}{ Item Type } & $\begin{array}{c}\text { Object- } \\
\text { Based }\end{array}$ & Perspective & $\begin{array}{c}\text { Both or } \\
\text { Neither }\end{array}$ \\
\hline Same/different & Human bodies & $18(75 \%)$ & $0(0 \%)$ & $6(25 \%)$ \\
& Animals & $22(91.7 \%)$ & $0(0 \%)$ & $2(8.3 \%)$ \\
& Inanimate objects & $22(91.7 \%)$ & $0(0 \%)$ & $2(8.3 \%)$ \\
Left/right & Human bodies & $5(21.7 \%)$ & $18(78.3 \%)$ & $0(0 \%)$ \\
& Animals & $9(26.1 \%)$ & $14(60.9 \%)$ & $3(13.0 \%)$ \\
& Inanimate objects & $9(26.1 \%)$ & $9(39.1 \%)$ & $8(34.8 \%)$ \\
\hline
\end{tabular}

Note-Percentages are given in parentheses. Row totals sum to $100 \%$. 
right task; a Fisher's exact test of independence showed this difference to be statistically significant $(p<.001)$. To focus on perspective transformations (the strategy of interest), we coded each response in the left/right task on the basis of whether it described a perspective transformation. This effectively combined the object-based transformation and "other" categories into a "not perspective transformation" category. A Fisher's exact test of independence indicated that the proportion of perspective transformation responses depended on item type $(p<.05)$, confirming that the use of perspective transformations was indeed most prevalent for images of human bodies and least prevalent for inanimate objects.

\section{Discussion}

Experiment 2 was designed to address the possibility that there were carryover effects associated with performing both the same/different and left/right tasks in succession. The RT results closely resembled those from Experiment 1, although the interactions of interest were only marginally significant in Experiment 2 . However, the introspective report data showed stronger patterns in Experiment 2. Participants who performed the left/right task reported more frequent use of perspective transformations when making judgments about bodies or animals than when making judgments about objects, as predicted. This suggests that participants' self-reports in Experiment 1 may have been constrained by the fact that they were collected after six experimental blocks involving two tasks and three stimulus sets. The validity and reliability of introspective reports have sometimes been called into question (Dunlap, 1912; Pylyshyn, 2003). Despite the existence of such criticism, we believe that self-report data, when considered within the context of other behavioral measures, can indeed provide useful converging evidence about the cognitive operations involved in performing spatial judgments. ${ }^{1}$

\section{GENERAL DISCUSSION}

We hypothesized that pictures of human bodies would selectively afford perspective transformations, in which participants transform their egocentric reference frame to bring it into alignment with that of a picture, whereas pictures of inanimate objects would selectively afford object-based transformations, in which participants transform the reference frame of a pictured object. This proposed dissociation was supported by the relations between stimulus orientation and RT, and by self-report measures. The RT effects were significant only in Experiment 1, and the self-report effects were present only in Experiment 2. Experiment 1 had greater sensitivity to detect RT effects, because all variables were manipulated within participants. Experiment 2 likely produced more reliable introspective reports because each participant performed only one of the two spatial tasks, eliminating the possibility of contamination across the two tasks. It must be noted, however, that the observed effects of animacy were small, which suggests that, to a certain extent, both object-based and perspective transformations are used to reason about animate and inanimate figures.

This study extends previous studies to a new class of stimuli: nonhuman animals. Animals are important because they share some, but not all, of the visuospatial and conceptual properties that distinguish human bodies from inanimate objects. Both are animate. However, people do many things that other animals do not do, and coordinating one's behavior with other people is likely more ecologically relevant than is coordinating one's behavior with nonhuman animals. The pattern observed here suggests that some combination of the shared and differing features matters for this type of spatial reasoning. Future research should include a systematic attempt to unpack which features do matter.

What is it that people are doing differently when reasoning about bodies rather than about objects? Zacks and Tversky (2005) proposed that pictures of bodies selectively encourage visuospatial reasoning strategies based on perspective transformations, whereas pictures of manipulable inanimate objects selectively encourage strategies based on object-based transformations. The present results are consistent with this proposal. According to this account, there are at least two potential explanations for the fact that nonhuman animal pictures produced responding intermediate between body pictures and object pictures. One possibility is that perspective transformations are evoked by the potential for interaction with a social agent. Human bodies should be strong cues for social interaction, and inanimate objects should be relatively poor cues. Animals should be intermediate, because people have some degree of social interaction with other species, such as pets, farm animals, and zoo animals, but are generally much less likely to participate in the sorts of interactions for which perspective transformations are important, such as coordinated action. Another possibility is that perspective transformations are evoked by figures that are animate - that is, figures that can move on their own. Animals might be less likely than bodies to evoke perspective transformations, because they are recognized less quickly or easily as being animate.

To tease apart these possibilities, one could independently manipulate participants' construals of stimuli as social actors or as animate beings. For example, to increase perceived animacy, one could use a video of each stimulus engaging in self-initiated motion. To reduce the perceived potential for social interaction while preserving animacy, one could present stimuli in a context that made them appear to be pictures transmitted from a remote location, such as from a security camera. Another way to characterize the effects of animacy on spatial reasoning would be to more fully explore the continuum of animacy. Inanimate objects vary in the degree to which they resemble living things in their visual properties and in the way that they move. A toy dog may resemble a real dog more or less closely, and machines such as robots and construction equipment may move in more or less human-like ways. This degree of similarity has been shown to influence the way in which an observer interacts with computer-generated animations 
and robots (Parise, Kiesler, Sproull, \& Waters, 1999). The present results replicated those of Zacks and Tversky (2005) for bodies and objects. However, in the present study, we observed relatively smaller differences in RT between these two item types. This may have been due to the constraints we imposed on the visual characteristics of the stimuli during the selection process. For example, the images of human bodies in our sample were uncharacteristically squat, whereas most natural pictures of humans depict them in an upright position. Had the visual properties of the images been allowed to vary more freely across types, there may well have been greater differences in task performance across the three item types.

\section{CONCLUSION}

In summary, we found that people performed spatial reasoning tasks differently with images of human bodies, animals, and inanimate objects, even after controlling for differences in visual characteristics of the stimulus images. These results indicate that category-level information about depicted figures can influence transformations of visuospatial representations during spatial reasoning. One possibility that is consistent with our results is that human-like visual stimuli are more likely to evoke transformations of one's egocentric reference frame, whereas manipulable inanimate stimuli are more likely to evoke transformations of the reference frame of the stimulus. This may have implications for the design of navigational interfaces and visualization tools. For example, users of GPS devices may find it easier to understand their current heading when it is represented by a human figure rather than by an automobile or an arrow. Visualization tools, such as those used in medical imaging or computer-aided design, could be improved by presenting body-relevant spatial cues. For example, a stylized human head pointing in a particular direction could be a more natural way to represent the orientation of a teleoperated surgical implement. Future work could examine whether life experiences, such as training animals or studying architecture, make it easier and more likely for some individuals to adopt novel spatial perspectives.

\section{AUTHOR NOTE}

The authors thank two anonymous reviewers for their comments and Laura D'Andrea for her assistance in experiment preparation and data collection. A.B.Y. was supported by a Science, Mathematics, and Research for Transformation (SMART) scholarship awarded by the United States Department of Defense. Address correspondence to A. B. Yu, Department of Psychology, Washington University, St. Louis, MO 63130 (e-mail: alfredyu@wustl.edu).

\section{REFERENCES}

Amorim, M.-A., IsAbleu, B., \& JARraya, M. (2006). Embodied spatial transformations: "Body analogy" for the mental rotation of objects. Journal of Experimental Psychology: General, 135, 327-347. doi:10 .1037/0096-3445.135.3.327

Bryant, D. J., TVERSKY, B., \& FrankLin, N. (1992). Internal and external spatial frameworks for representing described scenes. Journal of Memory \& Language, 31, 74-98. doi:10.1016/0749-596X(92)90006-J

Cohen, J., MacWhinney, B., Flatt, M., \& Provost, J. (1993). Psy-
Scope: An interactive graphic system for designing and controlling experiments in the psychology laboratory using Macintosh computers. Behavior Research Methods, Instruments, \& Computers, 25, 257-271.

Decety, J., \& Grèzes, J. (2006). The power of simulation: Imagining one's own and other's behavior. Brain Research, 1079, 4-14. doi:10.1016/j.brainres.2005.12.115

Downing, P. E., Jiang, Y., Shuman, M., \& Kanwisher, N. (2001). A cortical area selective for visual processing of the human body. Science, 293, 2470-2473. doi:10.1126/science. 1063414

DunLAP, K. (1912). Discussion: The case against introspection. Psychological Review, 19, 404-413. doi:10.1037/h0071571

Emmorey, K., Tversky, B., \& TAylor, H. A. (2000). Using space to describe space: Perspective in speech, sign, and gesture. Journal of Spatial Cognition \& Computation, 2, 157-180. doi:10.1023/ A: 1013118114571

Flavell, J. H., Flavell, E. R., Green, F. L., \& Wilcox, S. A. (1981). The development of three spatial perspective-taking rules. Child Development, 52, 356-358. doi:10.2307/1129250

Ganis, G., Keenan, J. P., Kosslyn, S. M., \& Pascual-Leone, A. (2000). Transcranial magnetic stimulation of primary motor cortex affects mental rotation. Cerebral Cortex, 10, 175-180. doi:10.1093/ cercor/10.2.175

HaRe, B., \& Tomasello, M. (2005). Human-like social skills in dogs? Trends in Cognitive Sciences, 9, 439-444. doi:10.1016/j.tics.2005 .07 .003

HaRris, I. M., \& Miniussi, C. (2003). Parietal lobe contribution to mental rotation demonstrated with rTMS. Journal of Cognitive Neuroscience, 15, 315-323. doi:10.1162/089892903321593054

Heptulla-Chatterjee, S., Freyd, J. J., \& Shiffrar, M. (1996). Configural processing in the perception of apparent biological motion. Journal of Experimental Psychology: Human Perception \& Performance, 22, 916-929. doi:10.1037/0096-1523.22.4.916

Huttenlocher, J., \& Presson, C. C. (1973). Mental rotation and the perspective problem. Cognitive Psychology, 4, 277-299. doi:10.1016/ 0010-0285(73)90015-7

KossLYN, S. M. (1980). Image and mind. Cambridge, MA: Harvard University Press.

Kozhevnikov, M., Motes, M. A., Rasch, B., \& Blajenkova, O. (2006). Perspective-taking vs. mental rotation transformations and how they predict spatial navigation performance. Applied Cognitive Psychology, 20, 397-417. doi:10.1002/acp.1192

Linde, C., \& Labov, W. (1975). Spatial networks as a site for the study of language and thought. Language, 51, 924-939.

McCloskey, M. (2001). Spatial representation in mind and brain. In B. Rapp (Ed.), The handbook of cognitive neuropsychology: What deficits reveal about the human mind (pp. 101-132). Philadelphia: Psychology Press.

Michelon, P., \& ZACKs, J. M. (2006). Two kinds of visual perspective taking. Perception \& Psychophysics, 68, 327-337.

Parise, S., Kiesler, S., Sproull, L., \& Waters, K. (1999). Cooperating with life-like interface agents. Computers in Human Behavior, 15, 123-142. doi:10.1016/S0747-5632(98)00035-1

PARsons, L. M. (1987). Imagined spatial transformation of one's body. Journal of Experimental Psychology: General, 116, 172-191. doi:10 .1037/0096-3445.116.2.172

Piaget, J., \& Inhelder, B. (1956). The child's conception of space. London: Routledge \& Kegan Paul.

Presson, C. C. (1982). Strategies in spatial reasoning. Journal of Experimental Psychology: Learning, Memory, \& Cognition, 8, 243-251. doi:10.1037/0278-7393.8.3.243

Pylyshyn,Z.W. (2002). Mental imagery: In search of a theory. Behavioral \& Brain Sciences, 25, 157-238. doi:10.1017/S0140525X02000043

Pylyshyn, Z. [W.] (2003). Return of the mental image: Are there really pictures in the brain? Trends in Cognitive Sciences, 7, 113-118. doi:10.1016/S1364-6613(03)00003-2

SCHOBER, M. F. (1993). Spatial perspective-taking in conversation. $\mathrm{Cog}$ nition, 47, 1-24. doi:10.1016/0010-0277(93)90060-9

SHEPARD, R. N. (1988). The role of transformations in spatial cognition. In J. Stiles-Davis, M. Kritchevsky, \& U. Bellugi (Eds.), Spatial cognition: Brain bases and development (pp. 81-110). Hillsdale, NJ: Erlbaum. 
SHEPARD, R. N., \& COOPER, L. A. (1982). Mental images and their transformations. Cambridge, MA: MIT Press.

Shepard, R. N., \& Metzler, J. (1971). Mental rotation of threedimensional objects. Science, 171, 701-703. doi:10.1126/science .171 .3972 .701

Traxler, M. J., \& Gernsbacher, M. A. (1993). Improving written communication through perspective-taking. Language \& Cognitive Processes, 8, 311-334. doi:10.1080/01690969308406958

TVersky, B., Lee, P., \& Mainwaring, S. (1999). Why do speakers mix perspectives? Spatial Cognition \& Computation, 1, 399-412. doi:10 .1023/A:1010091730257

Urgesi, C., Berlucchi, G., \& Aglioti, S. M. (2004). Magnetic stimulation of extrastriate body area impairs visual processing of nonfacial body parts. Current Biology, 14, 2130-2134. doi:10.1016/j.cub .2004 .11 .031

Vogeley, K., May, M., Ritzl, A., Falkai, P., Zilles, K., \& Fink, G. R. (2004). Neural correlates of first-person perspective as one constituent of human self-consciousness. Journal of Cognitive Neuroscience, 16, 817-827. doi:10.1162/089892904970799

Wraga, M., Creem, S. H., \& Proffitt, D. R. (2000). Updating displays after imagined object and viewer rotations. Journal of Experimental Psychology: Learning, Memory, \& Cognition, 26, 151-168. doi:10.1037/0278-7393.26.1.151

ZACKs, J. M. (2008). Neuroimaging studies of mental rotation: A metaanalysis and review. Journal of Cognitive Neuroscience, 20, 1-19. doi:10.1162/jocn.2008.20013

Zacks, J. M., Gilliam, F., \& Ojemann, J. G. (2003). Selective disturbance of mental rotation by cortical stimulation. Neuropsychologia, 41, 1659-1667. doi:10.1016/S0028-3932(03)00099-X

ZACKS, J. M., \& MiCHELON, P. (2005). Transformations of visuospatial images. Behavioral \& Cognitive Neuroscience Reviews, 4, 96-118. doi:10.1177/1534582305281085

Zacks, J. M., Mires, J., Tversky, B., \& Hazeltine, E. (2000). Mental spatial transformations of objects and perspective. Spatial Cognition \& Computation, 2, 315-332. doi:10.1023/A:1015584100204

Zacks, J. M., Ollinger, J. M., Sheridan, M. A., \& Tversky, B. (2002). A parametric study of mental spatial transformations of bodies. NeuroImage, 16, 857-872. doi:10.1006/nimg.2002.1129

Zacks, J. M., Rypma, B., Gabrieli, J. D. E., Tversky, B., \& Glover, G. H. (1999). Imagined transformations of bodies: An fMRI in- vestigation. Neuropsychologia, 37, 1029-1040. doi:10.1016/S0028 -3932(99)00012-3

ZACKs, J. M., \& TVERSKy, B. (2005). Multiple systems for spatial imagery: Transformations of objects and bodies. Spatial Cognition \& Computation, 5, 271-306. doi:10.1207/s15427633scc0504_1

Zacks, J. M., Vettel, J. M., \& Michelon, P. (2003). Imagined viewer and object rotations dissociated with event-related fMRI. Journal of Cognitive Neuroscience, 15, 1002-1018. doi:10.1162/ 089892903770007399

\section{NOTE}

1. Our paradigm was designed to distinguish between two spatial transformation-based strategies, but there remains the possibility that participants could have adopted other strategies that did not depend on spatial transformations. For example, the left/right task participants occasionally reported applying a rule that mapped left onto right and vice versa if the figure was upright and that mapped left onto left and vice versa if the figure was inverted. Such rule use was reported both in addition to performing a spatial transformation and as an alternative to performing a spatial transformation. In Experiment 1, 16 of 228 strategy reports mentioned such a rule; in Experiment 2, 28 of 141 reports mentioned such a rule. We repeated the RT analyses after excluding all participants who mentioned using a mapping rule; the pattern of data was unchanged. All the reported main effects, interactions, and post hoc comparisons remained statistically significant (with the one exception being the main effect of item type in Experiment 1). Thus, nontransformational strategies were used, although in the minority of cases, and it is unlikely that the RT patterns resulted from participants applying simple rules rather than performing spatial transformations.

\section{SUPPLEMENTAL MATERIALS}

A more in-depth discussion of two low-level visual characteristics, symmetry and skinniness, and their effects on task performance may be downloaded from http://mc.psychonomic-journals.org/content/ supplemental.

(Manuscript received July 27, 2009; revision accepted for publication March 14, 2010.) 